Received: 7 February 2017

Accepted: 8 May 2017

Published online: 21 June 2017

\title{
A note on the stationary
} distribution of stochastic SEIR epidemic model with saturated incidence rate

\author{
Qixing $\mathrm{Han}^{1}$, Liang Chen ${ }^{2} \&$ Daqing Jiang ${ }^{3,4}$
}

The stochastic SEIR infectious diseases model with saturated incidence rate is studied in this paper. By constructing appropriate Lyapunov functions, we show that there is a stationary distribution for the system and the ergodicity holds provided $R_{0}^{s}>1$. In particular, we improve the results obtained by previous studies greatly, condition in our Theorem is more concise and elegant.

The study of the epidemic models have long been and will continue to be one of the dominant themes in mathematical biology due to its importance at both understanding the spread and control of infectious diseases in a community. Many researchers have made a significant progress on $S I R$ models $^{1-6}$, where $S, I, R$ denote the fractions of the susceptibles, the infectives and the recovered hosts in the population respectively. SIR models assume the disease has no latent period. However, for some diseases, such as hepatitis B, AIDS, sometimes has to be passed before an infected individual becomes infectious. Therefore, an extra class, the class of exposed hosts $(E)$, should be added to the system, where $E$ denotes the fraction of the exposed population. The model is called SEIR (susceptible-exposed-infected-removed) model, and SEIR models were investigated by many researchers ${ }^{7-9}$.

On the other hand, the incidence rate palys an important role in the epidemics models. some authors employ the bilinear incidence rate $\beta S I^{10,11}$. After studying the cholera epidemic spread in Bari in 1973, Capasso and Serio $^{12}$ introduced a saturated incidence rate $g(I) S$ into epidemic models, where $g(I)$ tends to a saturation level when $I$ gets large, i.e.

$$
g(I) S=\frac{\beta I S}{1+\alpha I}
$$

where $\beta I$ measures the infection force of the disease and $\frac{1}{1+\alpha I}$ measures the inhibition effect from the behavioral change of the susceptible individuals when their number increases or from the crowding effect of the infective individuals. Then the SEIR model with a saturated incidence rate can be described as follows:

$$
\left\{\begin{aligned}
d S & =\left(\lambda-\frac{\beta S I}{1+\alpha I}-d_{S} S\right) d t \\
d E & =\left[\frac{\beta S I}{1+\alpha I}-\left(d_{E}+\theta\right) E\right] d t \\
d I & =\left[\theta E-\left(d_{I}+\delta+\gamma\right) I\right] d t \\
d R & =\left(\gamma I-d_{R} R\right) d t .
\end{aligned}\right.
$$

The parameters in the model are positive constants and summarized in the following:

${ }^{1}$ School of Mathematics, Changchun Normal University, Changchun, 130032, China. ${ }^{2}$ School of Mathematics and Statistics, Northeast Normal University, Changchun, 130024, China. ${ }^{3}$ College of Science, China University of Petroleum(East China), Qingdao, 266580, China. ${ }^{4}$ Nonlinear Analysis and Applied Mathematics (NAAM)-Research Group, King Abdulaziz University, Jeddah, Saudi Arabia. Correspondence and requests for materials should be addressed to L.C. (email: chen1234@nenu.edu.cn) or D.J. (email: daqingjiang2010@hotmail.com) 
$\lambda:$ the birth rate,

$d_{S}, d_{E}, d_{I}, d_{R}$ : natural death rates of $S, E, I, R$, respectively,

$\delta$ : additional disease caused rate suffered by the infectious individuals,

$\theta$ : the rate at which the exposed individuals become infectious,

$\gamma$ : the recovery rate of infective individuals.

Define the basic reproductive number $R_{0}=\frac{\lambda \beta \theta}{d_{S}\left(\theta+d_{E}\right)\left(d_{1}+\delta+\gamma\right)}$. The dynamical behavior of model (1.1) is as follows ref. 13:

If $R_{0}<1$, system (1.1) has a unique disease-free equilibrium $P_{0}=\left(\frac{\lambda}{d_{S}}, 0,0,0\right)$, which is a global attractor in the first octant.

If $R_{0}>1$, then model (1.1) has two equilibria, a disease-free equilibrium $P_{0}$ and an endemic equilibrium $P^{*}=\left\{S^{*}, E^{*}, I^{*}, R^{*}\right\} . P_{0}$ is unstable and $P^{*}$ is a global attractor in the interior of the first octant.

Some authors take stochastic perturbation into account when they investigate the epidemics system ${ }^{14-21}$. In this paper, we assume that the perturbation is of white noise type, that is, $d_{S} \rightarrow d_{S}+\sigma_{1} \dot{B}_{1}(t), d_{E} \rightarrow d_{E}+\sigma_{2} \dot{B}_{2}(t)$, $d_{I} \rightarrow d_{I}+\sigma_{3} \dot{B}_{3}(t), d_{R} \rightarrow d_{R}+\sigma_{1} \dot{B}_{4}(t)$, then we get the following stochastic system

$$
\left\{\begin{aligned}
d S & =\left(\lambda-\frac{\beta S I}{1+\alpha I}-d_{S} S\right) d t+\sigma_{1} S d B_{1}(t), \\
d E & =\left[\frac{\beta S I}{1+\alpha I}-\left(d_{E}+\theta\right) E\right] d t+\sigma_{2} E d B_{2}(t), \\
d I & =\left[\theta E-\left(d_{I}+\delta+\gamma\right) I\right] d t+\sigma_{3} I d B_{3}(t), \\
d R & =\left(\gamma I-d_{R} R\right) d t+\sigma_{4} R d B_{4}(t),
\end{aligned}\right.
$$

where $B_{1}(t), B_{2}(t), B_{3}(t), B_{4}(t)$ are standard one-dimensional independent Wiener processes, $\sigma_{i}\left(\sigma_{i}>0\right), i=1,2,3,4$ are the intensity of the white noise.

In ref. 17, Yang et al. show that there is a stationary distribution $\mu(\cdot)$ for system (1.2) and it has ergodic property provided the following conditions hold:

$$
\text { (H1) } R_{0}>1
$$

(H2) $\min \left\{k_{1} S^{*^{2}}, k_{2} E^{*^{2}}, k_{3} I^{*^{2}}, k_{4} R^{*^{2}}\right\}>\rho>0$,

where $\left(S^{*}, E^{*}, I^{*}, R^{*}\right)$ is the interior equilibrium of system of $(1.1), k_{1}=d_{S}-\sigma_{1}^{2}-\frac{\sigma_{1}^{2}\left(d_{E}+d_{S}+\theta\right)^{2} S^{*}}{d_{S}\left(d_{E}+\theta\right)}$, $k_{2}=\frac{d_{E}+\theta}{4}-\sigma_{2}^{2}, k_{3}=\frac{\left(d_{I}+\delta+\gamma\right)\left(d_{E}+\theta\right)}{8 \theta^{2}}\left(d_{I}+\delta+\gamma-4 \sigma_{3}^{2}\right), k_{4}=\frac{d_{R}\left(d_{I}+\delta+\gamma\right)^{2}\left(d_{E}+\theta\right)}{8 \theta^{2} \gamma^{2}}\left(d_{R}-2 \sigma_{4}^{2}\right)$, and

$$
\begin{aligned}
\rho= & \sigma_{1}^{2}\left[\frac{\left(d_{E}+d_{S}+\theta\right)^{2} S^{*^{3}}}{d_{S}\left(d_{E}+\theta\right)}+S^{*^{2}}+\frac{\beta S^{*} I^{*}}{d_{S}\left(1+\alpha^{*}\right)}\right] \\
& +\sigma_{2}^{2}\left[E^{*^{2}}+\frac{S^{*} E^{*}}{2}+\frac{\beta S^{*} E^{*} I^{*}}{2 d_{S}\left(1+\alpha^{*}\right)}\right] \\
& +\sigma_{3}^{2}\left[\frac{\left(d_{I}+\delta+\gamma\right)\left(d_{E}+\theta\right) I^{*^{2}}}{2 \theta^{2}}\right. \\
& \left.+\frac{S^{*} I^{*^{2}}\left(d_{E}+d_{S}+\theta\right)^{2}}{4 \theta d_{S}}\left(1+\frac{\beta}{d_{S}\left(1+\alpha^{*}\right)}\right)\right] \\
& +\frac{\sigma_{4}^{2} d_{R}\left(d_{I}+\delta+\gamma\right)^{2}\left(d_{E}+\theta\right) R^{* 2}}{4 \theta^{2} \gamma^{2}} .
\end{aligned}
$$

The main aim of this paper is to deal with the existence of stationary distribution of system (1.2). By construct new Lyapunov functions and rectangular set, instead of elliptical region, our results do not depend on the equilibrium $P^{*}$ of the deterministic system (1.1), which will improve the above result to a great extent.

\section{Ergodic Properties}

In order to show the existence of a stationary distribution, firstly, we cite a known result from ref. 22 as a lemma.

Let $X(t)$ be a homogeneous Markov Process in $E_{l}$ ( $E_{l}$ denotes $l$ dimensional Euclidean space), and is described by the following stochastic equation:

$$
d X(t)=b(X) d t+\sum_{r=1}^{k} g_{r}(X) d B_{r}(t) .
$$

The diffusion matrix is defined as follows: 


$$
\Lambda(x)=\left(\lambda_{i j}(x)\right), \quad \lambda_{i j}(x)=\sum_{r=1}^{k} g_{r}^{i}(x) g_{r}^{j}(x) .
$$

Lemma 2.1. The Markov process $X(t)$ has a unique ergodic stationary distribution $\mu(\cdot)$ if there exists a bounded domain $U \in E_{l}$ with regular boundary $\Gamma$ and

$A_{1}$ : there is a positive number $M$ such that $\sum_{i, j=1}^{l} \lambda_{i j}(x) \xi_{i} \xi_{j} \geq M|\xi|^{2}, x \in U, \xi \in R^{l}$,

$A_{2}$ : there exist a nonnegative $C^{2}-$ function $V$ such that $L V$ is negative for any $E_{I} \backslash U$. Then

$$
P_{x}\left\{\lim _{T \rightarrow \infty} \frac{1}{T} \int_{0}^{T} f(X(t)) d t=\int_{E_{l}} f(x) \mu(d x)\right\}=1
$$

for all $x \in E_{l}$, where $f(\cdot)$ is a function integrable with respect to the measure $\mu$.

Theorem 2.1. Assume that

$$
R_{0}^{s}:=\frac{\lambda \beta \theta}{\left(d_{S}+\frac{\sigma_{1}^{2}}{2}\right)\left(d_{E}+\theta+\frac{\sigma_{2}^{2}}{2}\right)\left(d_{I}+\delta+\gamma+\frac{\sigma_{3}^{2}}{2}\right)}>1,
$$

then for any initial value $(S(0), E(0), I(0), R(0)) \in R_{+}^{4}$, there is a stationary distribution $\mu(\cdot)$ for system (1.2) and the ergodicity holds.

Proof. First, we denote

$$
b:=3\left(d_{S}+\frac{\sigma_{1}^{2}}{2}\right)^{\frac{2}{3}}\left[\left(\frac{\lambda \beta \theta}{\left(d_{E}+\theta+\frac{\sigma_{2}^{2}}{2}\right)\left(d_{I}+\delta+r+\frac{\sigma_{3}^{2}}{2}\right)}\right)^{\frac{1}{3}}-\left(d_{S}+\frac{\sigma_{1}^{2}}{2}\right)^{\frac{1}{3}}\right] .
$$

It is easy to see that $b>0$. Constructing a $C^{2}$-function $Q: R_{+}^{4} \rightarrow R_{+}$in the following form

$$
\begin{aligned}
Q(S, E, I, R)= & p\left(-\log S-c_{1} \log E-c_{2} \log I\right)+(S+E+I+R)^{\rho+1} \\
& -\log S-\log E-\log R \\
:= & p V_{1}+V_{2}+V_{3}+V_{4}+V_{5},
\end{aligned}
$$

where

$$
c_{1}=\frac{d_{S}+\frac{\sigma_{1}^{2}}{2}}{d_{E}+\theta+\frac{\sigma_{2}^{2}}{2}}, \quad c_{2}=\frac{d_{S}+\frac{\sigma_{1}^{2}}{2}}{d_{I}+\delta+r+\frac{\sigma_{3}^{2}}{2}},
$$

$p$ and $\rho$ are constants satisfying the following condition respectively

$$
\begin{gathered}
-p b+A+d_{S}+\frac{\sigma_{1}^{2}}{2}+\left(d_{E}+\theta\right)+\frac{\sigma_{2}^{2}}{2}+d_{R}+\frac{\sigma_{4}^{2}}{2} \leq-2, \\
f:=\left(d_{S} \wedge d_{E} \wedge\left(d_{I}+\delta\right) \wedge d_{R}\right)-\frac{1}{2} \rho\left(\sigma_{1}^{2} \vee \sigma_{2}^{2} \vee \sigma_{3}^{2} \vee \sigma_{4}^{2}\right)>0,
\end{gathered}
$$

and $A$ is determined in the following proof. It is easy to check that

$$
\liminf _{k \rightarrow \infty,(S, E, I . R) \in R_{+}^{4} \backslash U_{k}} Q(S, E, I, R)=+\infty,
$$

where $U_{k}=\left(\frac{1}{k}, k\right) \times\left(\frac{1}{k}, k\right) \times\left(\frac{1}{k}, k\right) \times\left(\frac{1}{k}, k\right)$. Besides, $Q(S, E, I, R)$ is a continuous function. Hence, $Q(S, E, I$, $R$ ) must have a minimum point $\left(S_{0}, E_{0}, I_{0}, R_{0}\right)$ in the interior of $R_{+}^{4}$. Then we define a $C^{2}$ - and nonnegative function $V: R_{+}^{4} \rightarrow R_{+}$as following

$$
V(S, E, I, R)=Q(S, E, I, R)-Q\left(S_{0}, E_{0}, I_{0}, R_{0}\right) .
$$

According to Itô’s formula, we get

$$
\begin{aligned}
L V_{1}= & -\left(\frac{\lambda}{S}+\frac{c_{1} \beta S I}{(1+\alpha I) E}+\frac{c_{2} \theta E}{I}\right)+\frac{\beta I}{1+\alpha I}+d_{S}+c_{1}\left(d_{E}+\theta\right) \\
& +c_{2}\left(d_{I}+\delta+r\right)+\frac{\sigma_{1}^{2}+c_{1} \sigma_{2}^{2}+c_{2} \sigma_{3}^{2}}{2} .
\end{aligned}
$$


Applying inequality $a+b+c \geq 3 \sqrt[3]{a b c}, a, b, c>0$, yields

$$
\begin{aligned}
& L V_{1} \leq-3\left(\frac{c_{1} c_{2} \lambda \beta \theta}{1+\alpha I}\right)^{\frac{1}{3}}+\frac{\beta I}{1+\alpha I}+d_{S}+c_{1}\left(d_{E}+\theta\right) \\
& +c_{2}\left(d_{I}+\delta+r\right)+\frac{\sigma_{1}^{2}+c_{1} \sigma_{2}^{2}+c_{2} \sigma_{3}^{2}}{2} \\
& =-3\left[\frac{\lambda \beta \theta\left(d_{S}+\frac{\sigma_{1}^{2}}{2}\right)^{2}}{\left(d_{E}+\theta+\frac{\sigma_{2}^{2}}{2}\right)\left(d_{I}+\delta+r+\frac{\sigma_{3}^{2}}{2}\right)(1+\alpha I)}\right]^{\frac{1}{3}} \\
& +3\left(d_{S}+\frac{\sigma_{1}^{2}}{2}\right)+\frac{\beta I}{1+\alpha I} \\
& =-3\left(d_{S}+\frac{\sigma_{1}^{2}}{2}\right)^{\frac{2}{3}}\left[\left(\frac{\lambda \beta \theta}{\left(d_{E}+\theta+\frac{\sigma_{2}^{2}}{2}\right)\left(d_{I}+\delta+r+\frac{\sigma_{3}^{2}}{2}\right)(1+\alpha I)}\right)^{\frac{1}{3}}\right. \\
& \left.-\left(d_{S}+\frac{\sigma_{1}^{2}}{2}\right)^{\frac{1}{3}}\right]+\frac{\beta I}{1+\alpha I} \text {. }
\end{aligned}
$$

Similarly,

$$
\begin{aligned}
L V_{2}= & (\rho+1)(S+E+I+R)^{\rho}\left(\lambda-d_{s} S-d_{e} E-\left(d_{I}+\delta\right) I-d_{R} R\right) \\
& +\frac{1}{2} \rho(\rho+1)(S+E+I+R)^{\rho-1} \\
& \times\left(\sigma_{1}^{2} S^{2}+\sigma_{2}^{2} E^{2}+\sigma_{3}^{2} I^{2}+\sigma_{4}^{2} R^{2}\right) \\
\leq & (\rho+1)(S+E+I+R)^{\rho} \\
& \times\left[\left(\lambda-\left(d_{S} \wedge d_{E} \wedge\left(d_{I}+\delta\right) \wedge d_{R}\right)(S+E+I+R)\right)\right] \\
& +\frac{1}{2} \rho(\rho+1)(S+E+I+R)^{\rho+1}\left(\sigma_{1}^{2} \vee \sigma_{2}^{2} \vee \sigma_{3}^{2} \vee \sigma_{4}^{2}\right) \\
= & \lambda(\rho+1)(S+E+I+R)^{\rho} \\
& -(\rho+1)\left[\left(d_{S} \wedge d_{E} \wedge\left(d_{I}+\delta\right) \wedge d_{R}\right)\right. \\
& \left.-\frac{1}{2} \rho\left(\sigma_{1}^{2} \vee \sigma_{2}^{2} \vee \sigma_{3}^{2} \vee \sigma_{4}^{2}\right)\right](S+E+I+R)^{\rho+1} \\
\leq & A-\frac{1}{2}(\rho+1) f(S+E+I+R)^{\rho+1} \\
\leq & A-\frac{1}{2}(\rho+1) f\left(S^{\rho+1}+E^{\rho+1}+I^{\rho+1}+R^{\rho+1}\right),
\end{aligned}
$$

where

$$
\begin{aligned}
A= & \sup _{S+E+I+R \in(0, \infty)}\left\{\lambda(\rho+1)(S+E+I+R)^{\rho}\right. \\
& \left.-\frac{1}{2}(\rho+1) f(S+E+I+R)^{\rho+1}\right\}<\infty,
\end{aligned}
$$

and $f$ is defined in (2.3). We also obtain that

$$
\begin{gathered}
L V_{3}=-\frac{\lambda}{S}+\frac{\beta I}{1+\alpha I}+d_{S}+\frac{\sigma_{1}^{2}}{2}, \\
L V_{4}=-\frac{\beta S I}{(1+\alpha I) E}+\left(d_{E}+\theta\right)+\frac{\sigma_{2}^{2}}{2}
\end{gathered}
$$

and

$$
L V_{5}=-\frac{r I}{R}+d_{R}+\frac{\sigma_{4}^{2}}{2}
$$


Therefore

$$
\begin{aligned}
L V \leq & -3 p\left(d_{S}+\frac{\sigma_{1}^{2}}{2}\right)^{\frac{2}{3}}\left[\left(\frac{\lambda \beta \theta}{\left(d_{E}+\theta+\frac{\sigma_{2}^{2}}{2}\right)\left(d_{I}+\delta+r+\frac{\sigma_{3}^{2}}{2}\right)(1+\alpha I)}\right)^{\frac{1}{3}}\right. \\
& \left.-\left(d_{S}+\frac{\sigma_{1}^{2}}{2}\right)^{\frac{1}{3}}\right]+\frac{(p+1) \beta I}{1+\alpha I} \\
& -\frac{\beta S I}{(1+\alpha I) E}-\frac{1}{2}(\rho+1) f\left(S^{\rho+1}+E^{\rho+1}+I^{\rho+1}+R^{\rho+1}\right)-\frac{\lambda}{S}-\frac{r I}{R} \\
& +A+d_{S}+\frac{\sigma_{1}^{2}}{2}+\left(d_{E}+\theta\right)+\frac{\sigma_{2}^{2}}{2}+d_{R}+\frac{\sigma_{4}^{2}}{2} .
\end{aligned}
$$

Consider the following bounded subset

$$
D=\left\{\epsilon_{1} \leq S \leq \frac{1}{\epsilon_{1}}, \epsilon_{1} \leq I \leq \frac{1}{\epsilon_{1}}, \epsilon_{2} \leq E \leq \frac{1}{\epsilon_{2}}, \epsilon_{2} \leq R \leq \frac{1}{\epsilon_{2}}\right\},
$$

where $\epsilon_{1}, \epsilon_{2}>0$ are sufficiently small numbers satisfying the following conditions

$$
\begin{aligned}
& -p \hat{b}+(p+1) \beta \epsilon_{1}+A+d_{S}+\frac{\sigma_{1}^{2}}{2}+\left(d_{E}+\theta\right)+\frac{\sigma_{2}^{2}}{2}+d_{R}+\frac{\sigma_{4}^{2}}{2} \leq-1, \\
& -\frac{\lambda}{\epsilon_{1}}+A+d_{S}+\frac{(p+1) \beta}{\alpha}+\frac{\sigma_{1}^{2}}{2}+\left(d_{E}+\theta\right)+\frac{\sigma_{2}^{2}}{2}+d_{R}+\frac{\sigma_{4}^{2}}{2} \leq-1, \\
& -\frac{\beta}{\left(1+\alpha \epsilon_{1}\right) \epsilon_{1}}+3 p\left(d_{S}+\frac{\sigma_{1}^{2}}{2}\right)+\frac{(p+1) \beta}{\alpha}+A+d_{S}+\frac{\sigma_{1}^{2}}{2}+\left(d_{E}+\theta\right) \\
& +\frac{\sigma_{2}^{2}}{2}+d_{R}+\frac{\sigma_{4}^{2}}{2} \leq-1 \text {, } \\
& -\frac{r}{\epsilon_{1}^{2}}+3 p\left(d_{S}+\frac{\sigma_{1}^{2}}{2}\right)+\frac{(p+1) \beta}{\alpha}+A+d_{S}+\frac{\sigma_{1}^{2}}{2}+\left(d_{E}+\theta\right) \\
& +\frac{\sigma_{2}^{2}}{2}+d_{R}+\frac{\sigma_{4}^{2}}{2} \leq-1 \text {, } \\
& -\frac{(\rho+1) f}{2 \epsilon_{1}^{\rho+1}}+3 p\left(d_{S}+\frac{\sigma_{1}^{2}}{2}\right)+\frac{(p+1) \beta}{\alpha}+A+d_{S}+\frac{\sigma_{1}^{2}}{2}+\left(d_{E}+\theta\right) \\
& +\frac{\sigma_{2}^{2}}{2}+d_{R}+\frac{\sigma_{4}^{2}}{2} \leq-1 \text {, } \\
& -\frac{(\rho+1) f}{2 \epsilon_{2}^{\rho+1}}+3 p\left(d_{S}+\frac{\sigma_{1}^{2}}{2}\right)+\frac{(p+1) \beta}{\alpha}+A+d_{S}+\frac{\sigma_{1}^{2}}{2}+\left(d_{E}+\theta\right) \\
& +\frac{\sigma_{2}^{2}}{2}+d_{R}+\frac{\sigma_{4}^{2}}{2} \leq-1 \text {, }
\end{aligned}
$$

where

$$
\begin{aligned}
\hat{b}:= & -3\left(d_{S}+\frac{\sigma_{1}^{2}}{2}\right)^{\frac{2}{3}}\left[\left(\frac{\lambda \beta \theta}{\left(d_{E}+\theta+\frac{\sigma_{2}^{2}}{2}\right)\left(d_{I}+\delta+r+\frac{\sigma_{3}^{2}}{2}\right)\left(1+\alpha \epsilon_{1}\right)}\right)^{\frac{1}{3}}\right. \\
& \left.-\left(d_{S}+\frac{\sigma_{1}^{2}}{2}\right)^{\frac{1}{3}}\right]
\end{aligned}
$$

We note that for sufficiently small $\epsilon_{1}$, condition (2.9) holds due to (2.2). Then 


$$
R_{+}^{4} \backslash D=D_{1} \cup D_{2} \cup \cdots \cup D_{8},
$$

where

$$
\begin{aligned}
& D_{1}=\left\{(S, E, I, R) \in R_{+}^{4}, 0<S<\epsilon_{1}\right\}, \\
& D_{2}=\left\{(S, E, I, R) \in R_{+}^{4}, 0<I<\epsilon_{1}\right\}, \\
& D_{3}=\left\{(S, E, I, R) \in R_{+}^{4}, \quad S \geq \epsilon_{1}, I \geq \epsilon_{1}, 0<E<\epsilon_{2}\right\}, \\
& D_{4}=\left\{(S, E, I, R) \in R_{+}^{4}, I \geq \epsilon_{1}, 0<R<\varepsilon_{2}\right\}, \\
& D_{5}=\left\{(S, E, I, R) \in R_{+}^{4}, S>\frac{1}{\varepsilon_{1}}\right\}, \\
& D_{6}=\left\{(S, E, I, R) \in R_{+}^{4}, I>\frac{1}{\varepsilon_{1}}\right\}, \\
& D_{7}=\left\{(S, E, I, R) \in R_{+}^{4}, E>\frac{1}{\varepsilon_{2}}\right\}, \\
& D_{8}=\left\{(S, E, I, R) \in R_{+}^{4}, R>\frac{1}{\varepsilon_{2}}\right\} .
\end{aligned}
$$

Therefore we consider the following eight cases:

Case 1. If $(S, E, I, R) \in D_{1}$,

$$
\begin{aligned}
L V \leq & -\frac{\lambda}{S}+3 p\left(d_{S}+\frac{\sigma_{1}^{2}}{2}\right)+A+d_{S}+\frac{(p+1) \beta}{\alpha} \\
& +\frac{\sigma_{1}^{2}}{2}+\left(d_{E}+\theta\right)+\frac{\sigma_{2}^{2}}{2}+d_{R}+\frac{\sigma_{4}^{2}}{2} \\
\leq & -\frac{\lambda}{\epsilon_{1}}+3 p\left(d_{S}+\frac{\sigma_{1}^{2}}{2}\right)+A+d_{S}+\frac{(p+1) \beta}{\alpha} \\
& +\frac{\sigma_{1}^{2}}{2}+\left(d_{E}+\theta\right)+\frac{\sigma_{2}^{2}}{2}+d_{R}+\frac{\sigma_{4}^{2}}{2} .
\end{aligned}
$$

Then it follows from (2.10) that

$$
L V \leq-1 .
$$

Case 2. If $(S, E, I, R) \in D_{2}$, we have

$$
\begin{aligned}
L V \leq & -3 p\left(d_{S}+\frac{\sigma_{1}^{2}}{2}\right)^{\frac{2}{3}}\left[\left(\frac{\lambda \beta \theta}{\left(d_{E}+\theta+\frac{\sigma_{2}^{2}}{2}\right)\left(d_{I}+\delta+r+\frac{\sigma_{3}^{2}}{2}\right)(1+\alpha I)}\right)^{\frac{1}{3}}-\left(d_{S}+\frac{\sigma_{1}^{2}}{2}\right)^{\frac{1}{3}}\right] \\
& +(p+1) \beta I+A+d_{S}+\frac{\sigma_{1}^{2}}{2}+\left(d_{E}+\theta\right)+\frac{\sigma_{2}^{2}}{2}+d_{R}+\frac{\sigma_{4}^{2}}{2} \\
\leq & -p \hat{b}+(p+1) \beta \varepsilon_{1}+A+d_{S}+\frac{\sigma_{1}^{2}}{2}+\left(d_{E}+\theta\right)+\frac{\sigma_{2}^{2}}{2}+d_{R}+\frac{\sigma_{4}^{2}}{2} .
\end{aligned}
$$

Using condition (2.9), we obtain

$$
L V \leq-1 .
$$

Case 3. If $(S, E, I, R) \in D_{3}$, we obtain that

$$
\begin{aligned}
L V \leq & 3 p\left(d_{S}+\frac{\sigma_{1}^{2}}{2}\right)+\frac{(p+1) \beta}{\alpha}-\frac{\beta S I}{(1+\alpha I) E}+A \\
& +d_{S}+\frac{\sigma_{1}^{2}}{2}+\left(d_{E}+\theta\right)+\frac{\sigma_{2}^{2}}{2}+d_{R}+\frac{\sigma_{4}^{2}}{2} \\
\leq & -\frac{\beta \epsilon_{1}^{2}}{\left(1+\alpha \varepsilon_{1}\right) \varepsilon_{2}}+3 p\left(d_{S}+\frac{\sigma_{1}^{2}}{2}\right)+A+d_{S} \\
& +\frac{(p+1) \beta}{\alpha}+\frac{\sigma_{1}^{2}}{2}+\left(d_{E}+\theta\right)+\frac{\sigma_{2}^{2}}{2}+d_{R}+\frac{\sigma_{4}^{2}}{2} .
\end{aligned}
$$

Choosing 


$$
\epsilon_{2}=\epsilon_{1}^{3}
$$

yields

$$
\begin{aligned}
L V \leq & -\frac{\beta}{\left(1+\alpha \epsilon_{1}\right) \epsilon_{1}}+3 p\left(d_{S}+\frac{\sigma_{1}^{2}}{2}\right)+A+d_{S}+\frac{(p+1) \beta}{\alpha} \\
& +\frac{\sigma_{1}^{2}}{2}+\left(d_{E}+\theta\right)+\frac{\sigma_{2}^{2}}{2}+d_{R}+\frac{\sigma_{4}^{2}}{2} \\
\leq & -1 .
\end{aligned}
$$

The last inequality holds according to (2.11).

Case 4. If ( $S, E, I, R) \in D_{4}$, condition (2.12) combining with (2.15) gives

$$
\begin{aligned}
L V \leq & 3 p\left(d_{S}+\frac{\sigma_{1}^{2}}{2}\right)+\frac{(p+1) \beta}{\alpha}-\frac{r I}{R}+A+d_{S} \\
& +\frac{\sigma_{1}^{2}}{2}+\left(d_{E}+\theta\right)+\frac{\sigma_{2}^{2}}{2}+d_{R}+\frac{\sigma_{4}^{2}}{2} \\
\leq & -\frac{r \epsilon_{1}}{\epsilon_{2}}+3 p\left(d_{S}+\frac{\sigma_{1}^{2}}{2}\right)+A+d_{S}+\frac{(p+1) \beta}{\alpha} \\
& +\frac{\sigma_{1}^{2}}{2}+\left(d_{E}+\theta\right)+\frac{\sigma_{2}^{2}}{2}+d_{R}+\frac{\sigma_{4}^{2}}{2} \\
\leq & -\frac{r}{\epsilon_{1}^{2}}+3 p\left(d_{S}+\frac{\sigma_{1}^{2}}{2}\right)+A+d_{S}+\frac{(p+1) \beta}{\alpha} \\
& +\frac{\sigma_{1}^{2}}{2}+\left(d_{E}+\theta\right)+\frac{\sigma_{2}^{2}}{2}+d_{R}+\frac{\sigma_{4}^{2}}{2} \\
\leq & -1 .
\end{aligned}
$$

Case 5. If $(S, E, I, R) \in D_{5}$, we obtain that

$$
\begin{aligned}
L V \leq & 3 p\left(d_{S}+\frac{\sigma_{1}^{2}}{2}\right)+\frac{(p+1) \beta}{\alpha}-\frac{1}{2}(\rho+1) f S^{\rho+1}+A+d_{S} \\
& +\frac{\sigma_{1}^{2}}{2}+\left(d_{E}+\theta\right)+\frac{\sigma_{2}^{2}}{2}+d_{R}+\frac{\sigma_{4}^{2}}{2} \\
\leq & -\frac{(\rho+1) f}{2 \epsilon_{1}^{\rho+1}}+3 p\left(d_{S}+\frac{\sigma_{1}^{2}}{2}\right)+\frac{(p+1) \beta}{\alpha}+A \\
& +d_{S}+\frac{\sigma_{1}^{2}}{2}+\left(d_{E}+\theta\right)+\frac{\sigma_{2}^{2}}{2}+d_{R}+\frac{\sigma_{4}^{2}}{2},
\end{aligned}
$$

this together with (2.13), we derive that

$$
L V \leq-1 .
$$

Case 6. If $(S, E, I, R) \in D_{6}$,

$$
\begin{aligned}
L V \leq & 3 p\left(d_{S}+\frac{\sigma_{1}^{2}}{2}\right)+\frac{(p+1) \beta}{\alpha}-\frac{1}{2}(\rho+1) f I^{\rho+1}+A+d_{S} \\
& +\frac{\sigma_{1}^{2}}{2}+\left(d_{E}+\theta\right)+\frac{\sigma_{2}^{2}}{2}+d_{R}+\frac{\sigma_{4}^{2}}{2} \\
\leq & -\frac{(\rho+1) f}{2 \epsilon_{1}^{\rho+1}}+3 p\left(d_{S}+\frac{\sigma_{1}^{2}}{2}\right)+\frac{(p+1) \beta}{\alpha}+A+d_{S} \\
& +\frac{\sigma_{1}^{2}}{2}+\left(d_{E}+\theta\right)+\frac{\sigma_{2}^{2}}{2}+d_{R}+\frac{\sigma_{4}^{2}}{2} \\
\leq & -1,
\end{aligned}
$$

which follows from (2.13).

Case 7. If $(S, E, I, R) \in D_{7}$, it follows that 


$$
\begin{aligned}
L V \leq & 3 p\left(d_{S}+\frac{\sigma_{1}^{2}}{2}\right)+\frac{(p+1) \beta}{\alpha}-\frac{1}{2}(\rho+1) f E^{\rho+1}+A \\
& +d_{S}+\frac{\sigma_{1}^{2}}{2}+\left(d_{E}+\theta\right)+\frac{\sigma_{2}^{2}}{2}+d_{R}+\frac{\sigma_{4}^{2}}{2} \\
\leq & -\frac{(\rho+1) f}{2 \epsilon_{2}^{\rho+1}}+3 p\left(d_{S}+\frac{\sigma_{1}^{2}}{2}\right)+\frac{(p+1) \beta}{\alpha}+A+d_{S} \\
& +\frac{\sigma_{1}^{2}}{2}+\left(d_{E}+\theta\right)+\frac{\sigma_{2}^{2}}{2}+d_{R}+\frac{\sigma_{4}^{2}}{2},
\end{aligned}
$$

which together with (2.14) implies that

$$
L V \leq-1
$$

Case 8. If $(S, E, I, R) \in D_{8}$, we obtain that

$$
\begin{aligned}
L V \leq & 3 p\left(d_{S}+\frac{\sigma_{1}^{2}}{2}\right)+\frac{(p+1) \beta}{\alpha}-\frac{1}{2}(\rho+1) f R^{\rho+1}+A+d_{S} \\
& +\frac{\sigma_{1}^{2}}{2}+\left(d_{E}+\theta\right)+\frac{\sigma_{2}^{2}}{2}+d_{R}+\frac{\sigma_{4}^{2}}{2} \\
\leq & -\frac{(\rho+1) f}{2 \epsilon_{2}^{\rho+1}}+3 p\left(d_{S}+\frac{\sigma_{1}^{2}}{2}\right)+\frac{(p+1) \beta}{\alpha}+A+d_{S} \\
& +\frac{\sigma_{1}^{2}}{2}+\left(d_{E}+\theta\right)+\frac{\sigma_{2}^{2}}{2}+d_{R}+\frac{\sigma_{4}^{2}}{2} .
\end{aligned}
$$

Combining (2.14), yields

$$
L V \leq-1 .
$$

Based on the discussion of the above eight cases, we obtain

$$
L V \leq-1, \quad(S, E, I, R) \in R_{+}^{4} \backslash D .
$$

Therefore, $A_{2}$ in Lemma 2.1 is satisfied. In addition, $A_{1}$ is also satisfied (see ref. 17). Theorem 2.2 is proved according to Lemma 2.1.

Remark 2.1. Yang et al. ${ }^{17}$ have studied system (1.2), in Theorem 3.3 they show that under conditions (H1) and (H2), there is a stationary distribution $\mu(\cdot)$ for the system. Comparing with Theorem 2.2 in our investigation, we only need condition $R_{0}^{s}>1$, without other conditions imposed on the coefficients. That is to say, Theorem 2.2 in large improves Theorem 3.3 in ref. 17. Moreover, we see that if $\sigma_{i}=0(i=1,2,3,4)$, the above condition is reduced to $R_{0}>1$, which is the condition for globally asymptotically stable of endemic equilibrium $P^{*}$ of system (1.1). And $R_{0}^{s}$ is smaller than the basic reproduction number $R_{0}$ of system (1.1).

\section{References}

1. Kermack, W. O. \& McKendrick, A. G. Contribution to mathematical theory of epidemics. P. Roy. Soc. Lond. A Mat. 115, 700-721 (1927).

2. Pathak, S., Maiti, A. \& Samanta, G. P. Rich dynamics of an SIR epidemic model. Nonlinear Anal. 15, 71-81 (2010).

3. Anderson, R. M. \& May, R. M. Infectious Diseases of Humans: Dynamics and Control (Oxford University Press, Oxford, 1998).

4. Beretta, E. \& Takeuchi, Y. Convergence results in SIR epidemic model with varying population sizes. Nonlinear Anal. 28, 1909-1921 (1997).

5. Korobeinikov, A. Lyapunov functions and global stability for SIR, SIRS, and SIS epidemiological models. Appl. Math. Lett. 15, 955-960 (2002)

6. Ruan, S. G. \& Wang, E. D. Dynamical behavior of an epidemic model with a nonlinear incidence rate. J. Differential Equations 188, 135-163 (2003)

7. Li, M. Y. \& Muldowney, J. S. Global stability for the SEIR model in epidemiology. Math. Biosci. 125, 155-164 (1995).

8. Li, M. Y., Smith, H. L. \& Wang, L. C. Global dynamics of an SEIR epidemic model with vertical transmission. SIAM J. Appl. Math. 62, 58-69 (2001)

9. Gao, S., Chen, L. \& Teng, Z. Pulse vaccination of an SEIR epidemic model with time delay. Nonlinear Anal. Real World Appl. 9, 599-607 (2008)

10. Gabriela, M., Gomes, M., White, L. J. \& Medley, G. F. The reinfection threshold. J. Theor. Biol. 236, 111-113 (2005).

11. Wang, W. \& Ruan, S. Bifurcation in epidemic model with constant removal rate infectives. J. Math. Anal. Appl. 291, 775-793 (2004).

12. Capasso, V. \& Serio, G. A generalization of the Kermack-Mckendrick deterministic epidemic model. Math. Biosci. 42, 43-61 (1978).

13. Korobeinikov, A. Global properties of infectious disease models with nonlinear incidence. Bull. Math. Biol. 69, 1871-1886 (2007).

14. Lahrouz, A. \& Settati, A. Asymptotic properties of switching diffusion epidemic model with varying population size. Appl. Math. Comput. 219, 11134-11148 (2013).

15. Lahrouz, A. \& Settati, A. Necessary and sufficient condition for extinction and persistence of SIRS system with random perturbation. Appl. Math. Comput. 233, 10-19 (2014). 
16. Lin, Y. G. \& Jiang, D. Q. Threshold behavior in a stochastic SIS epidemic model with standard incidence. J. Dyn. Diff. Equat. 26, 1079-1094 (2014).

17. Yang, Q. S., Jiang, D. Q. \& Shi, N. Z. The ergodicity and extinction of stochastically perturbed SIR and SEIR epidemic models with saturated incidence. J. Math. Anal. Appl. 388, 248-271 (2012).

18. Yang, Q. S. \& Mao, X. R. Extinction and recurrence of multi-group SEIR epidemic models with stochastic perturbations. Nonlinear Anal. Real World Appl. 14, 1434-1456 (2013).

19. Liu, M., Bai, C. \& Wang, K. Asymptotic stability of a two-group stochastic SEIR model with infinite delays. Commun. Noninear. 19, 3444-3453 (2014).

20. Zhang, X. H. \& Wang, K. Stochastic SEIR model with jumps. Appl. Math. Lett. 26, 133-143 (2013).

21. Witbooi, P. J. Stability of an SEIR epidemic model with independent stochastic perturbations. Phys. A. 392, 4928-4936 (2013).

22. Has'minskii, R. Z. Stochastic Stability of Differential Equations. In: Monogr. Textb. Mech. Solids Fluids, vol. 7 (Sijthoff \& Noordhoff, Alphen aan den Rijn. Netherlands, 1980).

\section{Acknowledgements}

The work was supported by NSFC of China Grant (No.11371085), the Fundamental Research Funds for the Central Universities (No. 15CX08011A), Scientific Research Foundation of Jilin Department of Education (No. 2015356), STDP of Jilin Province (Grant No. 20150520052JH) and Natural Science Foundation of Changchun Normal University.

\section{Author Contributions}

Qixing Han and Liang Chen wrote the main manuscript text. Daqing Jiang suggested to investigate the problem mentioned in the manuscript and review the manuscript with Liang Chen who also revised some typos of the original manuscript.

\section{Additional Information}

Competing Interests: The authors declare that they have no competing interests.

Publisher's note: Springer Nature remains neutral with regard to jurisdictional claims in published maps and institutional affiliations.

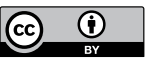

Open Access This article is licensed under a Creative Commons Attribution 4.0 International ( format, as long as you give appropriate credit to the original author(s) and the source, provide a link to the Creative Commons license, and indicate if changes were made. The images or other third party material in this article are included in the article's Creative Commons license, unless indicated otherwise in a credit line to the material. If material is not included in the article's Creative Commons license and your intended use is not permitted by statutory regulation or exceeds the permitted use, you will need to obtain permission directly from the copyright holder. To view a copy of this license, visit http://creativecommons.org/licenses/by/4.0/.

(C) The Author(s) 2017 